\title{
Thiết kế bộ điều khiển tự chinh thông số PiD trên cơ sở hệ luật
}

\author{
Lê Bá Dũng \\ Viẹn cong nghẹ thóng tin
}

\section{Mở đầu}

PID là bộ diều khiên được sử dụng rộng rài trong điều khiền các quá trình công nghiệp nói chung và trong điều khiĉn các quá trình công nghệ nởi riêng... . Ví dụ như trong điều khiền các robot công nghiệp, các robot dùng trong y học, các cơ cấu chấp hành thông minh. Trong thực tế khi điểu khiên, các thông số của hệ thống thường xuyên thay dồi, các bộ điều khiền PID thông thường không đáp ứng điều khiên được nhừng trường hợp như vậy. Sừ clụng bộ điều khiền PID thích nghi hay tự chinh đáp ứng được một số yêu cầu ờ trên, nhưng trong trường hợp này ta cùng phải xuất phát từ một yêu cầu thực tế là phải xây dựng mô hỉnh toán học của hệ thống thật chính xác. Thực tế nhiểu khi không đáp ứng đự̛̣ các yêu cầu đó bời lè hệ thống trong nhiĉ̀u trường hợp khó mà mô tả bời một mô hỉnh toán hiọc chînh xác hoặc hệ chịn tác động nhiểu mà ta không thể xác định được.... Thiết kế bộ điều khiền tự chinh (DKTC) thông số PID trên cơ sở hệ luật (HL) thóa mãn được nhừng yêu cầu trên. Trong [5] đà thiết kế bộ DKTC thông số PID trên cơ sở IIL được xây dựng theo sai lệch điểu khiên và sự thay đồi của sai lệch trong hai thời điềm cắt mầu liên tiếp nhau. Trong [6] cũng thiết kế bộ DKTC thông số PID trên cơ sở luật được xây dựng theo thay đồi của tín hiệu điểu khiền ờ thời điềm sau so với thời điềm trước. Nhîn chung các tác gia đều xây dựng các luật dưới dạng "if $\therefore$ and ... then ...".

Trong bài báo này nêu việc thiết kế bộ DKTC thông số PID trên cơ sờ HL sử dụng hệ mờ nhiều thông số (multivariable fuzzy system [\$]) với ba dầu vào là độ quá điểu khiển (overshoot) tỉ số (damper) xem hình 3 , thời gian đạt ổn định đầu 
tiên giá trị $2 \%$ tín hiệu chủ đạo (setlling time) và ba đâu ra là các hằng số $K, T_{i}, T d$ của bộ $K \mathrm{TC}$, các luật của HL này có cấu trúc dạng "if ... and ... and ... then ... and $\ldots$ and ...".

\section{Bộ DKTC thông số PID trên cơ sở hệ luật}

Kinh nghiệm trong linnh vực điểu khiên tự động rất là phong phú, những kinh nghiệm ấy càng nhiều lên trong những thập kỷ qua. Việc sử những kinh nghiệm ấy vào điểu khiển tự động là một hoài bão, mong muốn của các nhà làm việc trên linnh vực tự động. May mắn thay kỹ thuật máy tính phát triên, hệ chuyên gia ra đời đã giúp cho các nhà điêu khiên học ứng dụng được nó $[2,3]$ vào lính vực điêu khiên. Hơn thế nữa $[3,4]$ đã đưa những kinh nghiệm của các chuyên gia vào điêu khiên qua điều khiên mờ(fuzzy control) thông qua biến ngôn ngữ.

\subsection{Cấu trúc của bộ DKTC thông số PID trên cơ sớ HL}

Thông thường muốn thiết kế bộ ĐKTC thông số cân phải xây dựng một cơ cấu nhận dạng thông qua một phương pháp nhận dạng nào đấy. Trong thiết kế bộ ĐKTC thông số $\mathrm{PID}$ trên cơ sớ $\mathrm{HL}$ cơ cấu nhận dạng được xây dựng từ hành vi của đối tượng và những kinh nghiệm của các chuyên gia. Cơ cấu nhận dạng gồm các phần sau:

+ Cơ sờ tri thức

+ Cơ chế nhận biết (Pattern recognition engine)

+ Cơ chể suy diền (inference engine)

+ Cơ chế học (learning engine)

Quá trình nhận dạng thông số ở đây chính là thông qua các hành vi của đối tượng điều khiền, các thông tin từ đáp ứng ra của hệ hở cũng như hệ kín. Dó là việc tỉnh toán các giá trị ra của đáp ứng ra $y(t)$ và sai lệch điều khiền $e(t)$

$$
e(t)=r(t)-y(t)
$$

trong đó

$r(t)$ là tín hiệu chủ đạo

$y(t)$ là tín hiệu ra

$\epsilon(t)$ là sai lệch điều khiền.

Trong thực tế điều khiền các quá trình công nghệ, khi thay đổi tín hiệu chủ đạo (già sừ theo chiêu tăng) thì tín hiệu điểu khiên lúc đó đang ở giá trị ổn định $u(0)$ tăng lên giá trị $u(\max$ ) (già sư tăng liên tục) cho đến khi sai lệch $e(k)$ (sai lệch ở thời điềm $k$ ) đạt giá trị $e(k)=r(k)-y(k) \leq \epsilon^{*}$ thì $u(\max )$ sẽ được biến đồi thành $u(\min )$, thời gian cắt mẫu $T$ giữ giá trị $u(\min )$ cho đến khi tín hiệu ra $y(t)$ ôn định đến giá trị mới của tín hiệu chủ đạo $r(t)$, có nghía lúc đó $d y / d t=0$, xem hỉnh 1 và $u(\min )$ sẽ được đồi thành u (ồn định). Kinh'nghiệm của các nhà điểu khiển học [7] cho thấy 
$+e^{*}$ là quá nhò thì đầu ra có quá điồu khiền (overshoot)

+ nếu $\epsilon^{*}$ là quá lớn thì dầu ra chưa dạt dược giá trị ôn định (undershool)

+ nếu $T$ không chính xác thì $d y / d t \neq 0$

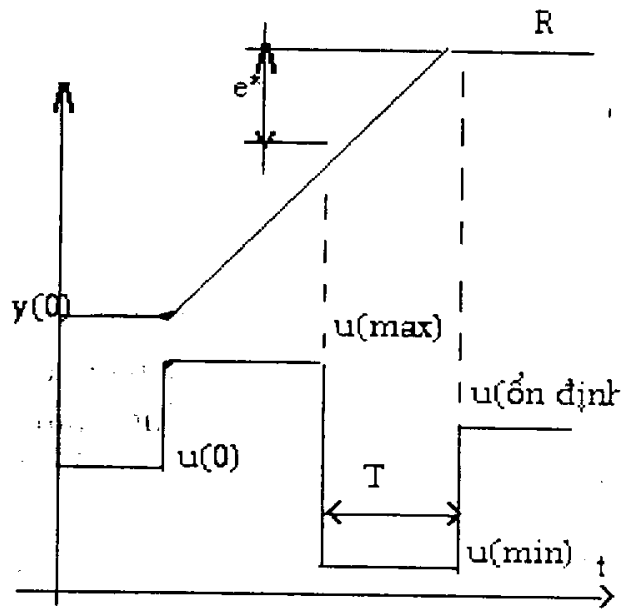

Hình 1. Sự thay dổi tín hiệu diều khiên khi thay dồi tín hiệu chui dạo

\subsection{Cơ só tri thức}

Thiết kế bộ DKTC thông số PID phài thoà màn uhững yêu câu cởbản sau:

+ Làm giảm sai lệch giữa tín hiệu ra và tín hiệu chủ đạo trên cơ sở chỉnh định các thông số cuia bộ điều chình PID

+ Đáp ưng yêu cầu của người thiết kế theo dáp ứng mong muốn (user specification)

+ Tự chinh được thông số khi có nhiêu tác động

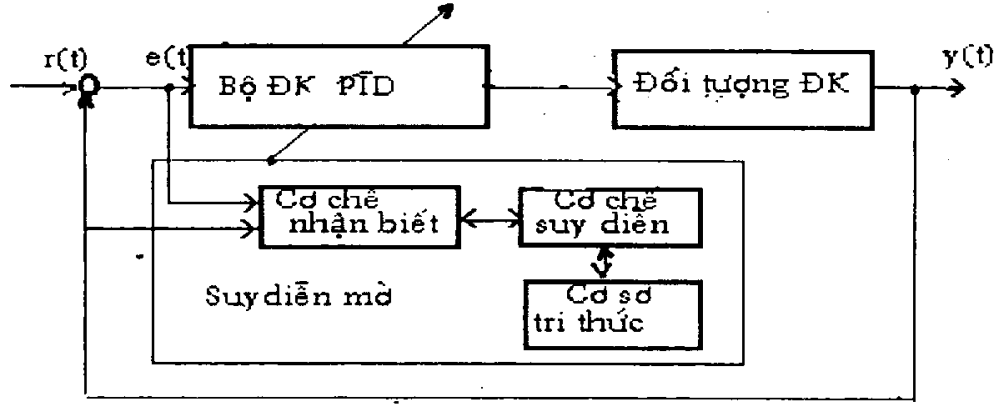

Hình y. Sơ đồ cấu trúc của bộ DKTC thông số PID

Phương trinhh bộ điểu khiển PID có dạng:

$$
u(k)=\kappa\left\{\epsilon(k)+T / A_{i} \sum e(k)+T d / T[e(k)-e(k-1)]\right\},
$$

ờ đây

$u(k)$ là tín hiệu điêu khiên ờ thời điêm $k$ 
$c(k)$ là sai lệch diều khiên ờ thời điềm $k$

Ti là hằng số tích phân

Td là hằng số vi phân

$T$ là thời gian cắt mẫu

$K$ là hệ số khuyếch đại.

Luật dùng đề chinh dịnh thông số cho bộ ĐKTC thông số PID trên cơ sở HL được mô tà dưới dạng

Nếu < tỉnh thế hay sụ kiện > thì < tác động >

< tác động > ở đây có nghià là thay đồi các hằng số $K, T i, T d$ trên cơ sở < tỉnh thế hay sự kiện > của trạng thái hay hành vi cuà đối tượng điểu khiên.

Nếu gọi OV là số lượng cuả quá trỉnh-điều khiên (amount overshoot), DP là (damping ratio), QR là tì số giữa (setlling time hiện hành và setlling time tại thời điềm trước) như đà định nghia ờ trên, thî hệ luật cho bộ DKTC thông số PID được xây dựng trên tri thức cuà các chuyên gía có dạng:

If $O V$ is PL and DP is PL then $K$ is PL and Ti is PL and $\mathrm{Td}$ is NL also,

If $O V$ is PL and DP is PM and QR is PL then $K$ is PL and Ti is NL and Td is $\mathrm{ZO}$ also,

If $\mathrm{OV}$ is PL and DP is PM and QR is $/ \mathrm{O}$ then $\mathrm{K}$ is $\mathrm{ZO}$ and $\mathrm{Ti}$ is PL and Td is $\mathrm{PL}$ also,

If $\mathrm{OV}$ is PL and DP is PM and $\mathrm{QR}$ is $\mathrm{NL}$ then $\mathrm{K}$ is $\mathrm{NL}$ and $\mathrm{Ti}$ is $\mathrm{PL}$ and $\mathrm{Td}$ is $\mathrm{PL}$ also,

If $\mathrm{OV}$ is PL and DP is $\mathrm{ZO}$ and QR is Pl, then $\mathrm{K}$ is PL and $\mathrm{Ti}$ is $\mathrm{ZO}$ and $\mathrm{Td}$ is 7O,

trong dó:

PL là positive large,

PM là positive medium.

ZO là zero,

NL là negative large.

Biêu diễn hàm thuộc cuà các tập mở $\mathrm{PL}, \mathrm{ZO}, \mathrm{NL}, \mathrm{PM}$, cho hằng số $K, T i, T d, Q P$

$\begin{array}{llllllllll} & -4 & -3 & -2 & -1 & 0 & +1 & +2 & +3 & +4 \\ \mathrm{PL} & 0 & 0 & 0 & 0 & 0 & 0 & .3 & .7 & 1 . \\ \mathrm{PM} & 0 & 0 & 0 & .3 & .7 & 1.0 & .7 & .3 & 0 \\ \mathrm{ZO} & 0 & 0 & .3 & .7 & 1.0 & .7 & .3 & 0 & 0 \\ \mathrm{NL} & 1.0 & .7 & .3 & 0 & 0 & 0 & 0 & 0 & 0\end{array}$

Biêu diên hàm thuộc của các tập mờ $\mathrm{PL}$ hằng số $\mathrm{OV}$

$\begin{array}{lllllllll}0 & 1 & 2 & 3 & 4 & 5 & 6 & 7 & 8\end{array}$


$\begin{array}{llllllllll}\mathrm{PL} & 0 & 0 & 0 & 0 & 0 & 0 & .3 & .7 & 1 .\end{array}$

Biêu diển hàm thuộc của các tập mờ $\mathrm{PL}, \mathrm{PM}, \mathrm{ZO}$ cho hằng số $\mathrm{DP}$

$\begin{array}{llllllllll} & .1 & .2 & .3 & .4 & .5 & .6 & .7 & .8 & .9 \\ \mathrm{PL} & 0 & 0 & 0 & 0 & 0 & 0 & .3 & .7 & 1 . \\ \mathrm{PM} & 0 & 0 & 0 & .3 & .7 & 1.0 & .7 & .3 & 0 \\ \mathrm{ZO} & 0 & 0 & .3 & .7 & 1.0 & .7 & .3 & 0 & 0\end{array}$

Bảng 1. Hàm thuộc của các biến ngôn ngữ

Hệ luật trên dược biều diên qua lý thuyêt tập mờ 1 rong đó quan hệ mờ được tỉnh theo $[8]$ :

$$
R=\bigvee_{i=1}^{5}\left(O r_{i} \wedge D P_{i} \wedge Q R_{i} \wedge K_{i} \wedge T i_{i} \wedge T d_{i}\right)
$$

$V$ ậy nếu ta đo được các giá trị vào $O V_{t}, D P_{t}, Q R_{t}$ ta có thể tính được dầu ra $y$ là vector

$$
\begin{gathered}
y_{t}=[K, T i, t d]^{T}=O V_{t} \cdot D P_{t} \cdot Q R_{t} \cdot R \\
y_{t}=\left\{\bigvee_{O V} O V_{t} \wedge \bigvee_{i=1}^{5} O V_{(i)}\right\} \wedge\left\{\{ \bigvee _ { D P } D P _ { t } \wedge \bigvee _ { i = 1 } ^ { 5 } D P _ { ( i ) } \wedge K _ { ( i ) } \} \wedge \left\{\left\{\bigvee_{Q R} Q R_{t} \wedge \bigvee_{i=1}^{5} Q R_{(i)} \wedge K_{(i)}\right\} \wedge\right.\right. \\
\left\{\bigvee_{O V} O V_{t} \wedge \bigvee_{i=1}^{5} O V_{(i)} \wedge T_{(i)}\right\} \wedge\left\{\bigvee_{D P} D P_{t} \wedge \bigvee_{i=1}^{5} D P_{(i)} \wedge T_{(i)}\right\} \wedge\left\{\left\{\bigvee_{Q R} Q R_{t} \wedge \bigvee_{i=1}^{5} Q R_{(i)} \wedge T d_{(i)}\right\} \wedge(5)\right. \\
\left\{\bigvee_{O V} O V_{t} \wedge \bigvee_{i=1}^{5} O V_{(i)} \wedge T d_{(i)}\right\} \wedge\left\{\bigvee_{D P} D P_{t} \wedge \bigvee_{i=1}^{5} D P_{(i)} \wedge T d_{(i)}\right\} \wedge\left\{\left\{\bigvee_{Q R} Q R_{t} \wedge \bigvee_{i=1}^{5} Q R_{(i)} \wedge T d_{(i)}\right\}\right.
\end{gathered}
$$

Các thành phần của vector $y_{t}$

$$
\begin{aligned}
K & =O V_{t} \circ R_{11} \bigwedge D P_{t} \circ R_{21} \bigwedge Q R_{t} \circ R_{31} \\
T_{i} & =O V_{t} \circ R_{12} \bigwedge D P_{t} \circ R_{22} \bigwedge Q R_{t} \circ R_{32} \\
T d & =O V_{t} \circ R_{13} \bigwedge D P_{t} \circ R_{23} \bigwedge Q R_{t} \circ R_{33}
\end{aligned}
$$

Trong dó

$$
\begin{aligned}
& R_{11}=\bigvee_{i=1}^{5} O V_{(i)} \wedge \cdot K_{(i)} ; R_{21}=\bigvee_{i=1}^{5} D P_{(i)} \wedge K_{(i)} ; R_{31}=\bigvee_{i=1}^{5} Q R_{(i)} \wedge K_{(i)} \\
& R_{12}=\bigvee_{i=1}^{5} O V_{(i)} \wedge T_{(i)} ; R_{22}=\bigvee_{i=1}^{5} D P_{(i)} \wedge T_{(i)} ; R_{32}=\bigvee_{i=1}^{5} Q R_{(i)} \wedge T_{(i)} \\
& R_{13}=\bigvee_{i=1}^{5} O V_{(i)} \wedge T d_{(i)} ; R_{23}=\bigvee_{i=1}^{5} D P_{(i)} \wedge T d_{(i)} ; R_{33}=\bigvee_{i=1}^{5} Q R_{(i)} \wedge T d_{(i)}
\end{aligned}
$$


và $\bigvee$ lấy giá trị max

$\wedge$ lấy giá trị min

o lấy giá trị maxmin

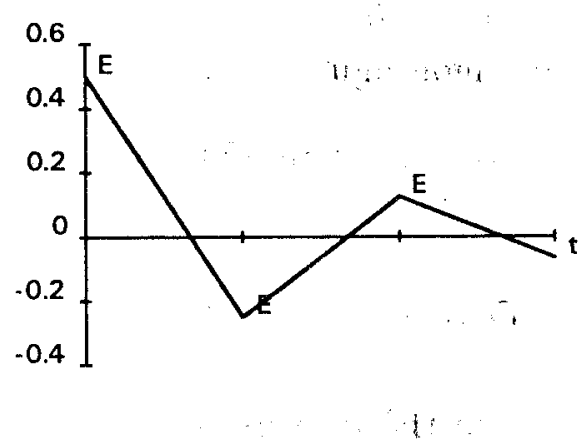

Hình 3 Tín hiĉu sai lêch diĉu khiên

$$
\text { Damping }=\frac{\mathrm{E} 0-\mathrm{E} 1}{\mathrm{E} 1-\mathrm{E} 2}
$$

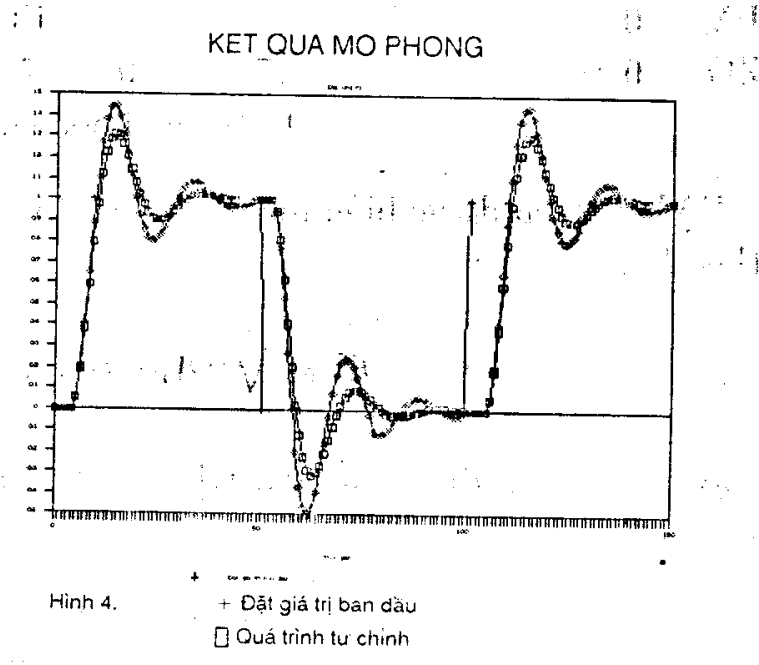

\subsection{Các bước thực hiện và kết quà mô phóng}

Đề bộ điều khiên có thề tự chinh các thông số $K, T i, T d$ cân phài thực hiện các bước sau:

- Dặt giá trị ban đầu $K, T i, T d$ cho quá trình điều khiền theo [9]

- Từ đáp ứng ra cuà hệ điều khiên, xác định các thông số như số lượng quá điều khiên, các đỉnh cuà quá điệu khiên chıra đạt, thời gian đạt ồ định đâu tiên (khoảng $2 \%$ tỉn hiệu chủ đạo)

- Mờ hóa các thông số đã được xác định thco các tập mò̀ L, PM, ...

- Tính toán các hằng số mờ $K, T i, T d$ theo hệ luật trên và chuyển đồi ra giá trị thực

- Thực hiện một tác động. $u(k)$ theo $(2)$ với các hằng số mới được tạo ra từ các bước trên

Kết quà mô phòng dược viết trên ngôn ngữ $\mathrm{C}$. Cho đối tượng có hàm truyền

$$
F(p)=\operatorname{Ki} \exp (-T ; p) /(T o p+1),
$$

với $T o=1 ; T_{z}=0.5 ; K=1$.

Hình 4 là kết quà mô phỏng cuả hệ. Qua các đáp ưng trên ta thấy quá trỉnh tự chinh các thông số được thực hiện sau khi đã ác định được các giá trị vê lượng 
quá điêu khiển, thời gian đạt ôn định. Từ đó cho phép ta đạt những yêu cầu vê thời gian đạt giá trị ồn định đầu tiên sao clıo ngăn nhất, độ sai lệch -y điểu khiền khi ồn định là không.

\section{Kết luận}

Bộ DKTC thông số PID trên cơ sở HL đã được thiết kế và kiêm nghiệm qua mô phồng. Kết quả trên cho thấy có thể sứ dụng thuật điểu khiên này vào các đối tượng công nghệ khác nhau cuả nhiều ngành công nghiệp.

\section{Tài liệu tham kháo}

1. Lê Bá Dũng \& Phạm Thượng Cát, Một số vấn dể co bản trong việc thiết kế bộ điểu khiên trên cơ sở hệ luạt, Tạp chí tin học và điêu khiển học số 1 1992, p. 1-9.

2. Astrom K.J., Anton J. \& Azen K.E., Expєrt Control, IFAC Congress, Budapest 1984.

3. Liu Limin, Knowledge-based Adaptive fuzzy Control, IFAC-ITAC, Singapore-90.

4. King P.J. \& Madami E.H., The application of fuzzy Control Systems to Industrial Processes, Automatica, v. 13, 1977, p. 235-242.

5. Lee K.B., Selp-tunning PID Controller: An Expert Systems Approach, IFACITAC, Singapore-90.

6. Walter H.B., Robert J. ..., Design of a Selp-Tunning Rule Based Controller for a Gasoline Refinery Catalytic Reformer, IEEE Trans. on Autom. Control, v. 15, 1990, n. 2, February.

7. Sripada N.R., Fisher D.G. \& Morris A.J., AI application for process and servo control, IEE proc. v. 134, 1987, July.

8. Some properties of Expert Control Systems, Approximate Reasoning in Expret Systems Elsevier Science Publ., North-Holland 198.5.

9. Liesleko J. ..., An Expert System for Tunning PID Controllers, IFAC-CAD in Cont rol Systems, Beijing, PRC 1988.

10. Phạm Thượng Cát, ..., Do và điểu khiên sư dụng máy vi tính, Sẽ xuất bản.

\section{Abstract \\ Some problems design-tunning PID controllers based on Rule-Based control \\ An implimentation of a selp-tunning PID Controller using Rule-besed fuzzy ex- pert systems approached is described in this paper. The algorithm uses fuzzy logic inferencing to adaptivity tune the parameters of the PID Controllers.}

